\title{
Effect of Thoracic Cava Obstruction on Response of Proximal Tubule Sodium Reabsorption to Saline Infusion*
}

\author{
William J. Cirksena, † John H. Dirks, and Robert W. Berliner with the \\ TECH NICAL ASSistance of Nordica Green \\ (From the Laboratory of Kidney and Electrolyte Metabolism of the National Heart Institute, \\ Bethesda, $M d$.)
}

We have recently demonstrated that in the normal dog infusion of isotonic or hypertonic saline results in marked depression of sodium reabsorption in the proximal tubule (1). This depression is independent of the effect of saline infusion on filtration rate and is not blocked by reduction of filtration rate. Such depression in proximal tubule reabsorption appears to account for the diuresis known, from the studies of De Wardener, Mills, Clapham, and Hayter $(2,3)$ and others $(4-7)$, to occur after saline infusion.

The present micropuncture study was undertaken to evaluate the possibility that sodium retention in states associated with edema formation might be related to failure of the normal decrease in proximal tubule sodium reabsorption to occur in response to salt loading. Reabsorption in the proximal tubule was evaluated by micropuncture techniques before and after acute partial obstruction of the thoracic inferior vena cava. Results indicate that the depression of proximal tubule sodium reabsorption that follows saline loading can be prevented and reversed by thoracic cava obstruction. This effect is independent of change in filtration rate or renal venous pressure.

\section{Methods}

Studies were performed on male and female mongrel dogs weighing 10 to $25 \mathrm{~kg}$. Dogs were rapidly anesthetized by intravenous injection of $25 \mathrm{mg}$ per $\mathrm{kg}$ sodium

* Submitted for publication August 16, 1965 ; accepted October 25, 1965.

Presented in part at the Fifty-seventh Annual Meeting of the American Society for Clinical Investigation, Atlantic City, N. J., May 3, 1965.

$\dagger$ Special Fellow, Office of the Surgeon General, Department of the Army, Washington, D. C.

Address requests for reprints to Dr. William J. Cirksena, Laboratory of Kidney and Electrolyte Metabolism, National Heart Institute, Bethesda, Md. 20014. thiopental (Pentothal sodium) ${ }^{1}$ given as a $2.5 \%$ solution in distilled water. Anesthesia was maintained with additional intravenous Pentothal as necessary. An endotracheal tube was inserted in all animals. Polyethylene catheters were inserted into a foreleg vein for saline infusion, a jugular vein for inulin infusion, a femoral vein for collection of venous blood samples and measurement of venous pressure, and occasionally a femoral artery for measurement of arterial pressure. The ureter of the right "control" kidney was catheterized after exposure through a suprapubic incision. The left kidney served as the experimental kidney and was exposed through a flank incision. The left ureter was catheterized and the kidney supported and prepared for micropuncture as described previously (1). Inulin in isotonic $\mathrm{NaCl}$ solution was infused at $1.07 \mathrm{ml}$ per minute in concentration sufficient to provide a plasma inulin level of approximately $100 \mathrm{mg}$ per $100 \mathrm{ml}$.

Tubule fluid was collected from surface proximal tubule convolutions in oil-filled capillary pipettes and each sampled convolution marked and identified for subsequent re-collection of fluid from the same convolution. The methods used in the collection and handling of samples and the identification of sampled convolutions for re-collection were the same as described in detail for a previous study (1).

During micropuncture phases of the experiments, 15minute urine collections were made from experimental kidneys and, with exceptions owing to technical problems, from the control kidneys. Blood samples were obtained at the midpoint of each urine collection period for determination of inulin clearance.

Tubule fluid was analyzed for inulin concentration by a microanthrone method described in detail previously (1). Inulin in plasma and urine was determined by the anthrone method of Führ, Kaczmarczyk, and Krüttgen (8). Tubule fluid to plasma (TF/P) inulin ratios were determined as a measure of fractional water and sodium reabsorption; the concentration in plasma was estimated by interpolation to the midpoint of the tubule fluid collection when there were changes in plasma level. Plasma and urine sodium concentrations were determined with a Baird flame photometer DB-5. Venous and arterial pressures were measured by means of a calibrated Sta-

1 Abbott Laboratories, North Chicago, IIl. 
tham P32AA transducer connected to a single channel Sanborn model 60-1300 recorder.

Saline loading in this study was accomplished by infusion of $1.0 \mathrm{ml}$ per $\mathrm{kg}$ per minute $0.9 \% \mathrm{NaCl}$ for 20 to 25 minutes, and then $0.5 \mathrm{ml}$ per $\mathrm{kg}$ per minute to the conclusion of the experiment. All animals were given 10 mg deoxycorticosterone acetate (Percorten acetate) 2 and $5 . U$ vasopressin tannate in oil (Pitressin tannate in oil) 3 intramuscularly 12 to 18 hours before the experiment. Five $\mathrm{mg}$ deoxycorticosterone in oil was given 30 minutes before the experiment. A priming dose of $1 \mathrm{mg}$ $9 \alpha$-fluorohydrocortisone 4 and $33 \mathrm{mU}$ per $\mathrm{kg}$ vasopressin (Pitressin) was given at the start of the experiment with continuous infusion of $2 \mu \mathrm{g}$ per minute $9 \alpha$-fluorohydrocortisone and $100 \mathrm{mU}$ per $\mathrm{kg}$ per hour vasopressin throughout the experiment.

The effect of acute partial obstruction of the thoracic inferior vena cava (TIVC) on the proximal tubule response to saline loading was assessed in nine dogs. Control collections of tubule fluid were made during hydropenia. The inferior vena cava was then obstructed to raise venous pressure in the lower inferior vena cava 100 to $150 \mathrm{~mm}$ water above control levels. This was done in three experiments by tightening a ligature placed around the vena cava through a thoracotomy incision before beginning the micropuncture phase of the experiments. The resultant pneumothorax was evacuated through a thoracotomy tube connected to an underwater drain. In the remainder of the experiments, the TIVC was obstructed by inflation of a balloon catheter introduced through a femoral vein and positioned, with aid of fluoroscopy, in the vena cava just above the diaphragm. In most experiments, isotonic saline infusion was started at the time of obstruction of the vena cava and continued for 1 hour before fluid was again collected from the same proximal convolutions. Previous studies (1) demonstrated that depression in proximal tubule sodium reabsorption occurred within 1 hour of saline infusion in normal dogs. Tubule fluid was subsequently collected from the same convolutions after release of obstruction and establishment of diuresis during. continued saline infusion. In the earliest experiments, an attempt was made to obtain collections of tubule fluid after TIVC obstruction but before starting infusion of saline. However, collections of tubule fluid adequate for analysis could only rarely be made during TIVC obstruction without saline infusion because of marked reduction in flow in the tubules and relatively unstable physiologic condition of the animals. Attempts at such collections were abandoned in later experiments of the series.

The effect of elevation of renal venous pressure on the response of reabsorption in the proximal tubule to saline loading was evaluated in three dogs by partial obstruction of the abdominal inferior vena cava (AIVC) above the renal veins but below the diaphragm. AIVC obstruction to raise venous pressure measured in the lower

2 Ciba Pharmaceutical Co., Summit, N. J.

3 Parke, Davis, and Co., Detroit, Mich.

4 Upjohn, Kalamazoo, Mich. inferior vena cava 150 to $250 \mathrm{~mm}$ water above control levels was again accomplished by inflation of a balloon catheter that was positioned by palpation through the flank incision. The position of the catheter was confirmed at the conclusion of the experiments. Tubule fluid was collected from the same proximal convolutions during control hydropenia, during saline infusion with obstruction of the AIVC, and after release of cava obstruction during continued saline infusion.

\section{Results}

Control studies. Results of 63 paired collections involving repeated sampling of the same proximal convolutions during continued hydropenia have been reported previously (1). The presentation of these same data has been modified here to conform to the method of presentation of this study. The values in column 3 of Table IV have been obtained by dividing the final $\mathrm{TF} / \mathrm{P}$ ratio by the initial ratio for each sample pair. A value of 1.0 therefore indicates no mean change in $\mathrm{TF} / \mathrm{P}$ ratios between collections. Data have been calculated and presented similarly for all phases of this study. The data obtained on repeated collection during continued hydropenia (hydropenia controls) are included in this Table. The mean ratio of final to initial $\mathrm{TF} / \mathrm{P}$ ratios for these hydropenia controls, $0.97 \pm 0.013$, differs from 1.00 to only a minor, albeit statistically significant $(p=0.03)$, extent. These results indicate that repeated sampling of the same proximal convolution during hydropenia has only a trivial effect on fractional reabsorption.

A comparison of $\mathrm{TF} / \mathrm{P}$ ratios from the same convolution during control hydropenia and during saline diuresis with reduced filtration rate due to renal artery clamping reported previously (1) shows that the depression of proximal tubule fractional reabsorption of sodium and water that occurs with saline loading is not prevented by large reductions in filtration rate. The mean ratio of final to initial $T F / P$ ratios was $0.79 \pm$ 0.018 . This was significantly different from hydropenia controls, but not significantly different from saline loading alone compared with hydropenia $(0.84 \pm 0.020)$ reported previously (1), despite a mean reduction in filtration rate of $44.7 \%$. In studies to be reported (9), it has also been demonstrated that reduction of filtration rate by renal artery clamping during continued hydropenia does not alter proximal tubule frac- 
TABLE I

Thoracic inferior vena cava obstruction; detailed protocol*

\begin{tabular}{|c|c|c|c|c|c|c|c|c|c|c|c|}
\hline \multirow{2}{*}{$\underset{\text { time }}{\text { Elapsed }}$} & \multicolumn{2}{|c|}{ Urine flow } & \multicolumn{2}{|c|}{$\mathrm{UNaV}_{\mathrm{Na}}$} & \multicolumn{2}{|c|}{ Plasma } & \multicolumn{2}{|c|}{ Inulin clearance } & \multicolumn{2}{|c|}{ Tubule fluid } & \multirow[b]{2}{*}{$\begin{array}{l}\text { Inulin } \\
\text { TF/P }\end{array}$} \\
\hline & $\mathbf{E}$ & C & $\mathrm{E}$ & $\vec{C}$ & Inulin & $\overline{\text { Sodium }}$ & E & C & $\begin{array}{l}\text { Sample } \\
\text { collected }\end{array}$ & Inulin & \\
\hline minutes & \multicolumn{2}{|c|}{$m l / m i n$} & \multicolumn{2}{|c|}{$\mu E q / \min$} & $m g / m l$ & $m E q / L$ & \multicolumn{2}{|c|}{$m l / \min$} & \multicolumn{3}{|c|}{$m g / m l$} \\
\hline 15 & 0.53 & 0.68 & 39 & 58 & 1.47 & 142 & 28.0 & 30.8 & 1at & 203 & 1.38 \\
\hline 60 & 0.36 & 0.51 & 24 & 42 & 1.53 & 142 & 27.4 & 30.2 & $3 a$ & 285 & 1.87 \\
\hline 75 & 0.42 & 0.63 & 31 & 57 & 2.61 & 143 & 27.9 & 30.6 & $4 a$ & 270 & 1.74 \\
\hline 158 & \multicolumn{11}{|c|}{ TIVC partially obstructed by tightening ligature, venous pressure kept $100 \mathrm{~mm} \mathrm{H}_{2} \mathrm{O}$ above control level } \\
\hline 188 & 0.13 & $\ddagger$ & 1 & $\ddagger$ & 2.73 & 142 & 5.0 & $\ddagger$ & $3 \mathrm{~b}$ & 780 & 2.85 \\
\hline 189 & \multicolumn{11}{|c|}{$0.9 \% \mathrm{NaCl}$ infused $18 \mathrm{ml}$ per minute for 21 minutes, then $9.25 \mathrm{ml}$ per minute } \\
\hline \multirow[t]{2}{*}{315} & \multirow[t]{2}{*}{0.42} & & \multirow{2}{*}{\multicolumn{2}{|c|}{17}} & 2.56 & 146 & \multirow{2}{*}{\multicolumn{2}{|c|}{19.3}} & $4 c$ & 630 & 2.47 \\
\hline & & & & & & & & & $1 c$ & 482 & 1.90 \\
\hline 330 & 0.44 & & 17. & & 2.38 & 145 & 20.5 & & $3 c$ & 500 & 2.00 \\
\hline 341 & \multicolumn{11}{|c|}{$\begin{array}{l}\text { TIVC obstruction removed; venous pressure returned to control level; saline infusion continued at } 9.25 \\
\text { ml per minute }\end{array}$} \\
\hline \multirow[t]{2}{*}{372} & \multirow[t]{2}{*}{3.33} & & \multirow[t]{2}{*}{222} & & 3.12 & 145 & \multirow[t]{2}{*}{20.1} & & $3 d$ & 409 & 1.31 \\
\hline & & & & & & & & & $1 d$ & 360 & 1.16 \\
\hline 387 & 3.80 & & 267 & & 3.08 & 145 & 20.5 & & $4 d$ & 400 & 1.29 \\
\hline
\end{tabular}

*Abbreviations: $\mathrm{E}=$ experimental kidney, $\mathrm{C}=$ control kidney, $\mathrm{U}_{\mathrm{Na}} \mathrm{V}=$ excreted sodium, $\mathrm{TF} / \mathrm{P}=$ tubule fluid to plasma ratio, and TIVC $=$ thoracic inferior vena cava.

$\dagger \mathrm{a}, \mathrm{b}, \mathrm{c}, \mathrm{d}=$ consecutive collections from same tubule segment.

$\ddagger$ Catheter in control kidney lost.

tional reabsorption (Table IV, hydropenia clamp vs. hydropenia). Thus, reduced filtration rate alone does not affect proximal tubule fractional reabsorption or prevent the usual response to saline loading.

Thoracic vena cava obstruction. After TIVC obstruction, kidney volume was generally reduced grossly, suggesting that the reduction in renal perfusion was at least as significant as the elevation in renal venous pressure. In two experiments in which the results were not different from others in the series, blood pressure was monitored and did not change after TIVC obstruction with saline infusion. In all experiments but one, collections were made during saline infusion alone after release of TIVC obstruction. Table I shows a detailed protocol of one such experiment. In one experiment, this order of experimental conditions was reversed. Table II summarizes the changes in inulin $\mathrm{TF} / \mathrm{P}$ in this experiment during saline infusion alone and during obstruction of the TIVC with continued saline infusion. Results were not significantly different in this experiment from others in the group, and they have been analyzed together. Figure 1 summarizes the effect of acute obstruction of the TIVC during saline loading in nine dogs. The Figure presents a comparison of inulin TF/P ratios during saline infusion with TIVC obstruction with ratios from the same proximal convolutions during control hydropenia. $\mathrm{TF} / \mathrm{P}$ ratios remained essentially unchanged or increased variably during TIVC obstruction despite saline loading. The mean ratio of final to initial $\mathrm{TF} / \mathrm{P}$ ratios was $1.22 \pm 0.058$. This is a significant increase compared with hydropenia controls and is in sharp contrast to previous re-

TABLE II

Effect of thoracic cava obstruction on inulin $T F / P$

\begin{tabular}{cccc}
\hline \hline & $\begin{array}{c}\text { Inulin } \\
\text { TF/P } \\
\text { during } \\
\text { hydropenia }\end{array}$ & $\begin{array}{c}\text { Inulin } \\
\text { TF/P } \\
\text { during } \\
\text { saline } \\
\text { infusion } \\
\text { alone }\end{array}$ & $\begin{array}{c}\text { Inulin } \\
\text { TF/P } \\
\text { during } \\
\text { saline } \\
\text { administra- } \\
\text { tion with } \\
\text { TIVC ob- } \\
\text { struction }\end{array}$ \\
\hline 1 & 1.28 & 1.49 & 1.71 \\
2 & 1.63 & 1.12 & \\
3 & 1.29 & 1.12 & 2.06 \\
4 & 1.40 & 1.07 & 1.66 \\
5 & 1.44 & 1.10 & 2.24 \\
6 & 1.20 & 1.00 & 1.78 \\
7 & 1.29 & 1.03 & 1.04 \\
\hline
\end{tabular}




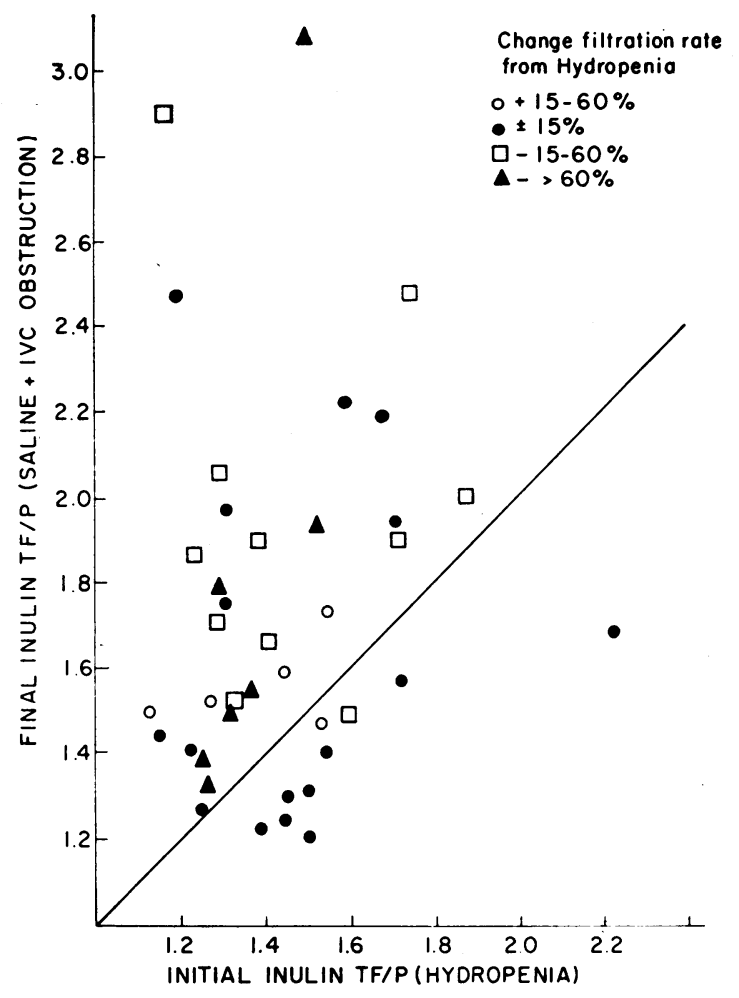

Fig. 1. EFFeCt OF THORACIC INFERIOR VENA CAVA (TIVC) OBSTRUCTION DURING ISOTONIC SALINE INFUSION IN NINE DOGS ON RATIO OF INULIN CONCENTRATION IN PROXIMAL TUBULE FLUID to THAT IN PLASMa (TF/P). Symbols indicate the extent of reduction of glomerular filtration rate (GFR).

sults of saline infusion without TIVC obstruction $(0.84 \pm 0.020)$ or saline infusion with reduced filtration rate due to renal artery clamping $(0.79 \pm$ 0.012 ) (1). The symbols used in Figure 1 indicate the changes in filtration rate that occurred during TIVC obstruction; these changes ranged from essentially zero to a reduction as great as $95 \%$. There was a mean decrease of $30 \%$ in filtration rate. The largest changes in $\mathrm{TF} / \mathrm{P}$ ratios occurred with the largest reductions in filtration rate, although large changes in filtration rate also accompanied small changes in $\mathrm{TF} / \mathrm{P}$ ratios. It is likely that both the changes in filtration rate and in fractional reabsorption result from the hemodynamic alterations that follow TIVC obstruction. However, as control experiments have shown, filtration rate change alone does not alter proximal $\mathrm{TF} / \mathrm{P}$ ratios, and reduction of filtration rate does not prevent the response of the proximal tubule to saline loading. These results indicate that the
TABLE III

Effect of abdominal cava obstruction on inulin $T F / P$

\begin{tabular}{|c|c|c|c|}
\hline Tubule & $\begin{array}{c}\text { Inulin } \\
\text { TF/P } \\
\text { during } \\
\text { hydropenia }\end{array}$ & $\begin{array}{c}\text { Inulin } \\
\text { TF/P } \\
\text { during } \\
\text { saline } \\
\text { plus AIVC* } \\
\text { obstruction }\end{array}$ & $\begin{array}{l}\text { Inulin } \\
\text { TF/P } \\
\text { during } \\
\text { saline } \\
\text { alone af ter } \\
\text { release of } \\
\text { obstruction }\end{array}$ \\
\hline 1 & 1.82 & 1.83 & 1.54 \\
\hline 2 & 1.89 & 1.62 & 1.77 \\
\hline 3 & 2.40 & 2.36 & 3.26 \\
\hline 4 & 2.54 & 1.56 & \\
\hline 5 & 1.99 & 1.61 & 1.61 \\
\hline 6 & 2.26 & 1.89 & 1.60 \\
\hline
\end{tabular}

* AIVC $=$ abdominal inferior vena cava.

increase in proximal tubule reabsorption occurring in these TIVC obstruction experiments does not result from changes in filtration rate, but is related in some other way to obstruction of the vena cava.

The relative unimportance of changes in filtration rate in the observed effects is further substantiated by results obtained after release of TIVC obstruction. In Figure 2 the $\mathrm{TF} / \mathrm{P}$ ratios during saline infusion after release of TIVC obstruction are compared with ratios during saline infusion with obstruction. Symbols indicate the change in filtration rate occurring between collections. Filtration rate increased significantly in some instances but remained essentially unchanged or decreased further in other instances after release of obstruction. Despite these variable changes in filtration rate, $T F / P$ ratios were uniformly lower after release of obstruction than ra-

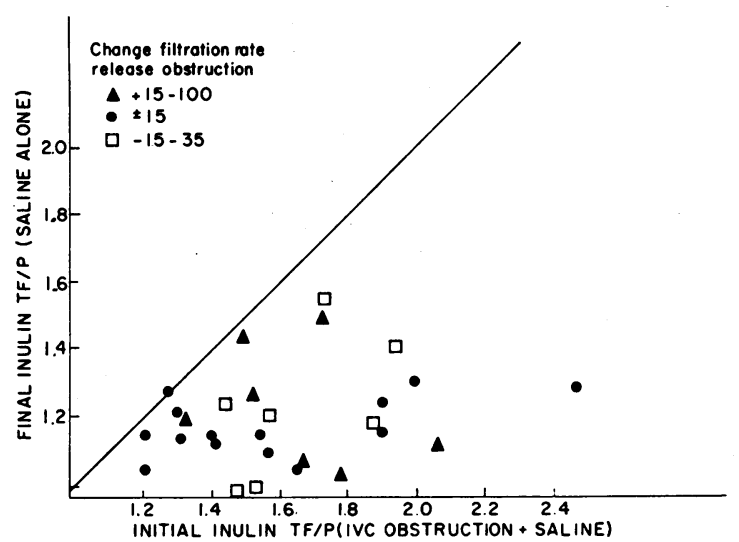

Fig. 2. EFFEct ON PROXIMAL INULIN TF/P OF RELEASE OF TIVC OBSTRUCTION WITH CONTINUED ISOTONIC SALINE INFUSION IN EIGHT DOGS. Symbols indicate the extent of change of GFR. 


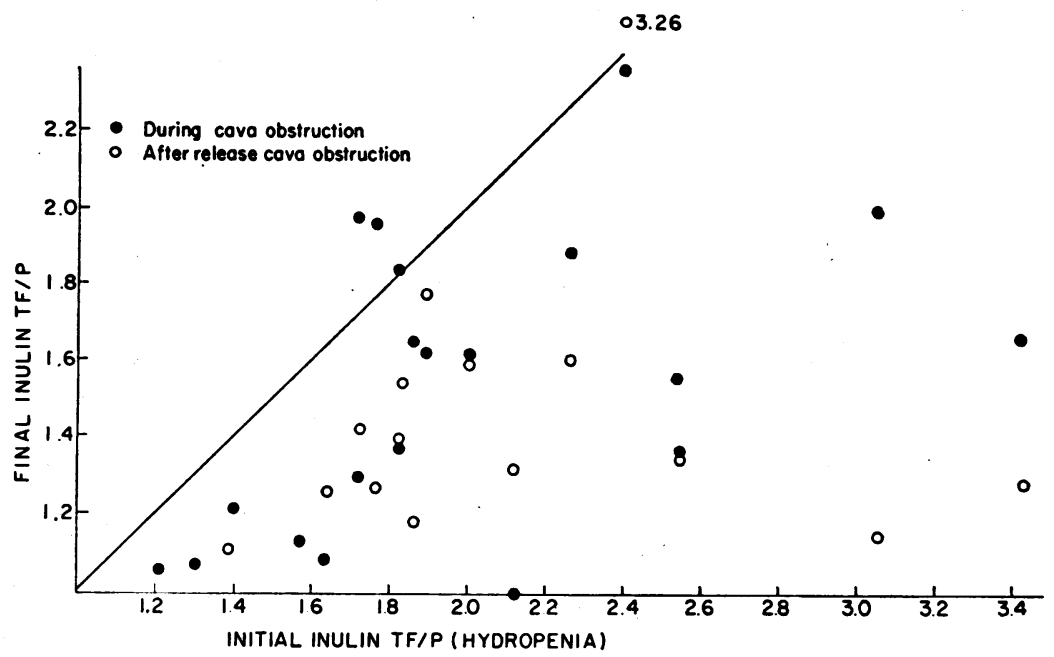

Fig. 3. EFfect on PROXIMAL INULIN TF/P OF ABDominal inferior vena CAVA (AIVC) OBSTRUCTION DURING ISOTONIC SALINE INFUSION IN THREE DOGS. (O) Compare AIVC obstruction plus saline with control hydropenia. (O) Compare saline infusion alone after release of AIVC obstruction with control hydropenia.

tios from the same convolutions during obstruction. The mean ratio of final to initial (during hydropenia) $\mathrm{TF} / \mathrm{P}$ ratios was $0.82 \pm 0.028$, significantly different from hydropenia controls and comparable to changes reported previously (1) with saline infusion without intervening cava obstruction. After release of TIVC obstruction, kidney volume increased grossly and diuresis occurred.
Abdominal vena cava obstruction. Table III shows results of a typical experiment in which renal venous pressure was elevated by partial obstruction of the AIVC. TF/P ratios during control hydropenia, during saline loading with AIVC obstruction, and during saline infusion after release of obstruction are shown. Closed circles in Figure 3 show results of 20 paired collections

TABLE IV

Summary of data on inulin $T F / P$ and $G F R^{*}$

\begin{tabular}{|c|c|c|c|c|}
\hline Experiment & & $\begin{array}{c}\text { Dogs } \\
\text { Sample } \\
\text { pairs }\end{array}$ & $\begin{array}{l}\text { Inulin TF/P } \\
\text { (final/initial } \\
\quad \pm \mathrm{SE})\end{array}$ & $\begin{array}{c}\text { Mean } \\
\text { change } \\
\text { in GFR }\end{array}$ \\
\hline Hydropenia control $\dagger$ & & $7-63$ & $0.97 \pm 0.013$ & $\begin{array}{r}\text { per cent } \\
+4.7 \ddagger\end{array}$ \\
\hline $\begin{array}{l}\text { Hydropenia plus renal artery } \\
\text { clamping vs. hydropenia }\end{array}$ & & $7-43$ & $1.00 \pm 0.024$ & $-50.1 \|$ \\
\hline $\begin{array}{l}\text { TIVC obstruction plus saline } \\
\text { vs. hydropenia }\end{array}$ & & $9-40$ & $1.22 \pm 0.058$ & $-29.8 \ddagger$ \\
\hline $\begin{array}{l}\text { Saline after release of TIVC } \\
\text { obstruction vs. hydropenia }\end{array}$ & 1 & $8-28$ & $0.82 \pm 0.028$ & $-15.0 \ddagger$ \\
\hline $\begin{array}{l}\text { AIVC obstruction plus } \\
\text { saline vs. hydropenia }\end{array}$ & & $3-20$ & $0.79 \pm 0.042$ & $+1.0 \ddagger$ \\
\hline $\begin{array}{l}\text { Saline after release of AIVC } \\
\text { obstruction vs. hydropenia }\end{array}$ & & $3-15$ & $0.74 \pm 0.061$ & $-0.2 \ddagger$ \\
\hline
\end{tabular}

* GFR = glomerular filtration rate.

† Recalculated from data previously reported (1).

¥ Per cent change in experimental kidney inulin clearance during recollection periods.

(9).

Per cent decrease in experimental kidney inulin clearance compared to control kidney and corrected for any difference between experimental and control kidneys during control periods. 
TABLE V

Percentage of filtered sodium excreted

\begin{tabular}{lll}
\hline & $\begin{array}{c}\text { Experi- } \\
\text { mental } \\
\text { kidney }\end{array}$ & $\begin{array}{c}\text { Control } \\
\text { kidney }\end{array}$ \\
\hline
\end{tabular}

TIVC obstruction

\begin{tabular}{lll}
$\begin{array}{l}\text { Control } \\
\text { Obstruction + saline }\end{array}$ & 1.1 & 1.4 \\
$\begin{array}{l}\text { Saline alone after release of } \\
\text { obstruction }\end{array}$ & 1.2 & 1.0 \\
$\quad$ & 5.4 & 5.3 \\
IVC obstruction & & \\
$\begin{array}{l}\text { Control } \\
\begin{array}{l}\text { Obstruction + saline } \\
\text { Saline alone after release of }\end{array}\end{array}$ & 0.1 & 0.6 \\
$\quad$ obstruction & 3.1 & 3.0 \\
\hline
\end{tabular}

comparing $\mathrm{TF} / \mathrm{P}$ ratios during saline infusion with abdominal cava obstruction with values during control hydropenia before obstruction. All but a few ratios fell during saline infusion despite AIVC obstruction sufficient to raise venous pressure 150 to $250 \mathrm{~mm}$ water above the control level. The ratio of final to initial TF/P ratios was 0.79 \pm 0.042 and in marked contrast to results with TIVC obstruction (1.22 \pm 0.058$)$. Open circles in Figure 3 indicate the relationship of TF/P ratios during saline infusion after release of AIVC obstruction to ratios from the same convolutions during control hydropenia. The ratio of final to initial $\mathrm{TF} / \mathrm{P}$ ratios was $0.74 \pm 0.061$, not different from saline infusion during TIVC obstruction. These results indicate that marked elevation of renal venous pressure alone does not prevent the depression of sodium reabsorption in the proximal tubule that follows saline loading, and that the results obtained with TIVC obstruction are not due to the effect of the latter procedure on renal venous pressure.

The results described above are summarized in Table IV. The effects of TIVC and AIVC obstruction on the fraction of filtered sodium excreted are summarized in Table V. There was no rise in sodium excretion after saline loading with TIVC obstruction. Natriuresis occurred after release of obstruction. AIVC obstruction did not prevent the usual natriuresis after saline loading.

\section{Discussion}

In a previous study (1), it has been shown that a marked depression of fractional reabsorption of sodium and water in the proximal convoluted tubule occurs after the administration of saline to normal dogs. The present series of experiments, in which the same re-collection micropuncture techniques were used, indicates that this depression of reabsorption in the proximal tubule can be prevented and reversed by partial obstruction of the thoracic inferior vena cava. Obstruction of the TIVC has been shown to prevent the diuresis and increase in sodium excretion that normally follow loading with saline (2-7).

Since TIVC obstruction resulted in both a reduction of glomerular filtration rate and an increase of renal venous pressure, the effects of these changes on fractional reabsorption in the proximal tubule require separate consideration. Filtration rate was reduced by a mean of $30 \%$ (range, 0 to $95 \%$ ) after TIVC obstruction in the present experiments. In a previous study, a mean reduction of filtration rate of $42.3 \%$ (range, 20 to $71 \%$ ) in dogs undergoing saline diuresis was produced by partial clamping of the renal artery. The reduced fractional reabsorption of filtrate in the proximal tubule that had developed in response to saline infusion was unaffected by the change in filtration rate. We have also found that fractional reabsorption in the proximal tubule in the hydropenic dog remains unchanged when glomerular filtration is reduced (mean reduction $50 \%$ ) by renal artery clamping. It thus seems highly unlikely that the failure of fractional reabsorption to decrease in response to saline loading in the presence of TIVC obstruction is a consequence of a diminished rate of glomerular filtration. This inference is further supported by the results obtained after release of the TIVC obstruction. Release of the obstruction had variable effects on filtration rate; in some instances filtration rate decreased further, whereas in others it increased. Regardless of the direction or magnitude of the change in filtration rate, fractional reabsorption of filtrate decreased in all instances. It is concluded, therefore, that the effect of TIVC obstruction on sodium reabsorption is not mediated by the decreased filtration rate.

Experiments in which the abdominal inferior vena cava was obstructed were performed to evaluate the effect of elevated renal venous pressure. Elevation of renal venous pressure by AIVC obstruction did not prevent the depression of frac- 
tional reabsorption after saline loading. The mean decrease in $\mathrm{TF} / \mathrm{P}$ ratio, $21 \%$, was comparable to that produced by saline infusion without AIVC obstruction. There was no significant further decrease after release of obstruction. Thus, the effect of TIVC obstruction on fractional reabsorption does not appear to be mediated by elevated renal venous pressure.

Our findings with respect to sodium excretion are in accord with the studies in saline-loaded dogs with chronic TIVC ligation of Davis and associates (10-13) and Levinsky and Lalone (14). Davis, Howell, and Southworth (10) showed that dogs with TIVC constriction continued to accumulate ascites for weeks to months during steroid administration, but failed to retain sodium after release of cava ligation despite massive steroid administration. Similar results with the kidney transplanted to the neck excluded the influence of the renal nerves or alterations in renal venous pressure in this response to cava ligation (12). The recent report of Levinsky and Lalone (14) has ruled out reduced filtration rate as a necessity for sodium retention in TIVC-constricted dogs by showing failure of natriuresis after saline loading despite large increases in filtration rate. The present micropuncture study suggests that sodium retention after acute TIVC obstruction can be accounted for by failure of the normal decrease in fractional sodium reabsorption in the proximal tubule to occur after saline loading. De Wardener and co-workers $(2,3)$ suggested that changing levels of a humoral agent other than aldosterone could account for the natriuresis they found after intravenous saline infusion. Davis and co-workers (13) also suggested that either a humoral agent or some as yet unidentified change in renal function mediates the change resulting in sodium retention in TIVC-constricted dogs. Our micropuncture observations suggest that if such a factor exists, its effect is exerted by alteration of fractional reabsorption in the proximal tubule.

The results could reflect the effects of a hormone that depresses sodium reabsorption. However, the observed results could also be explained by the formation of a salt-retaining substance not of adrenocortical origin, the secretion of which is suppressed and stimulated during saline loading and TIVC obstruction, respectively (2). De Wardener and his associates (2) and Lichardus and Pearce (15) have demonstrated that natriuresis occurs in isolated kidneys perfused in situ with blood from saline-loaded donors and donors infused with washed red cells suspended in bovine albumin-saline solution, respectively. Both groups concluded that a humoral agent was responsible for the renal sodium loss. Failure to obtain augmented sodium excretion and the occurrence of an increase in sodium reabsorption by the proximal tubule in TIVC-constricted dogs may, then, result from failure of the stimulus to, or interference with the mechanism of, the secretion of such a humoral factor. The nature and site of origin of any such humoral agent and the stimulus to its production have not been established. It is not unlikely that the expansion of extracellular fluid volume that follows saline loading provides the stimulus. The differing effects of thoracic and abdominal cava obstruction on proximal tubule reabsorption suggest that the differing hemodynamic consequences of such obstruction on hepatic venous or central venous volume or cardiac output may be involved in stimulating and suppressing production of such a humoral factor.

Our results with acute TIVC obstruction differ from the acute studies of Levinsky and Lalone (14) in that they found natriuresis with saline loading in dogs with acute TIVC ligation similar to that with AIVC ligation. The reasons for this difference are unclear but could be related to the degree of vena cava obstruction which, in the present experiments, produced a severe hemodynamic disturbance that frequently included considerable drops in the rate of glomerular filtration.

These studies show that fractional reabsorption of sodium by the proximal tubule may vary over a wide range. Saline infusion decreases fractional sodium reabsorption. TIVC obstruction increases fractional sodium reabsorption despite saline infusion. Alterations in filtration rate, renal venous pressure, or circulating levels of adrenal steroids or vasopressin do not account for these changes in fractional reabsorption in the proximal tubule. The relation of these observations to the mechanism of edema formation in clinical disorders depends on the extent to which the experimental model of acute TIVC obstruction simulates the hemodynamic or other alterations that occur in these states. If the experimental observations are applicable to the clinical situations. failure of na- 
triuresis in states associated with salt retention and edema can be accounted for by an altered response in proximal tubule fractional reabsorption of sodium. The precise intermediary mechanisms involved await further clarification.

\section{Summary}

1) The effect of acute partial obstruction of the thoracic inferior vena cava (TIVC) on the response of the proximal tubule to isotonic saline infusion has been evaluated in dogs loaded with steroid and vasopressin. Micropuncture collection from the same proximal convolutions during hydropenia and during saline infusion with TIVC obstruction was carried out.

2) Despite the infusion of saline, inulin tubule fluid to plasma ratios ( $\mathrm{TF} / \mathrm{P}$ ) increased significantly (mean 22\%) after TIVC obstruction, above values found in hydropenia. Natriuresis and diuresis did not occur. There was a fall in glomerular filtration rate (GFR) averaging $30 \%$.

3) After release of TIVC obstruction natriuresis and diuresis ensued despite variable changes in GFR, and inulin TF/P decreased significantly in all instances to a level averaging $18 \%$ below that found in hydropenia.

4) Elevation of renal venous pressure by acute partial obstruction of the abdominal inferior vena cava (AIVC) did not prevent natriuresis and diuresis after isotonic saline infusion, and inulin $\mathrm{TF} / \mathrm{P}$ fell significantly (mean $21 \%$ ) from the values found in hydropenia. There was no further appreciable change with release of AIVC obstruction.

5) These studies indicate that acute partial TIVC obstruction prevents and reverses the depression of proximal tubule sodium reabsorption that follows saline loading, and that this effect is independent of changes in filtration rate, renal venous pressure, or circulating levels of adrenal steroid or vasopressin.

\section{References}

1. Dirks, J. H., W. J. Cirksena, and R. W. Berliner. The effect of saline infusion on sodium reabsorption by the proximal tubule of the dog. J. clin. Invest. 1965, 44, 1160.

2. De Wardener, H. E., I. H. Mills, W. F. Clapham, and C. J. Hayter. Studies on the efferent mecha- nism of the sodium diuresis which follows the administration of intravenous saline in the dog. Clin. Sci. 1961, 21, 249.

3. Mills, I. H., H. E. De Wardener, C. J. Hayter, and W. F. Clapham. Studies on the afferent mechanism of the sodium chloride diuresis which follows intravenous saline in the dog. Clin. Sci. 1961, 21, 259.

4. Levinsky, N. G., and R. C. Lalone. The mechanism of sodium diuresis after saline infusion in the dog. J. clin. Invest. 1963, 42, 1261.

5. Blythe, W. B., and L. G. Welt. Dissociation between filtered load of sodium and its rate of excretion in the urine. J. clin. Invest. 1963, 42, 1491.

6. Rector, F. C., Jr., G. Van Giesen, F. Kiil, and D. W. Seldin. Influence of expansion of extracellular volume on tubular reabsorption of sodium independent of changes in glomerular filtration rate and aldosterone activity. J. clin. Invest. 1964, 43, 341.

7. Earley, L. E., and R. M. Friedler. Observations on the mechanism of decreased tubular reabsorption of sodium and water during saline loading. J. clin. Invest. 1964, 43, 1928.

8. Führ, J., J. Kaczmarczyk, and C. D. Krüttgen. Eine einfache colorimetrische Methode zur Inulinbestimmung für Nieren-clearance-untersuchungen bei Stoffwechselgesunden and Diabetikern. Klin. Wschr. 1955, 33, 729.

9. Cirksena, W. J., J. H. Dirks, and R. W. Berliner. Unpublished data.

10. Davis, J. O., D. S. Howell, and J. L. Southworth. Mechanisms of fluid and electrolyte retention in experimental preparations in dogs. III. Effect of adrenalectomy and subsequent desoxycorticosterone acetate administration on ascites formation. Circulat. Res. 1953, 1, 260.

11. Davis, J. O., D. S. Howell, M. J. Goodkind, and R. E. Hyatt. Accumulation of ascites during maintenance of adrenalectomized dogs with thoracic inferior vena cava constriction on a high sodium diet without hormone therapy. Amer. J. Physiol. 1956, 185, 230.

12. Carpenter, C. C. J., J. O. Davis, J. E. Holman, C. R. Ayers, and R. C. Bahn. Studies on the response of the transplanted kidney and the transplanted adrenal gland to thoracic inferior vena caval constriction. J. clin. Invest. 1961, 40, 196.

13. Davis, J. O., J. E. Holman, C. C. J. Carpenter, J. Urquhart, and J. T. Higgins, Jr. An extraadrenal factor essential for chronic renal saline retention in presence of increased sodium-retaining hormone. Circulat. Res. 1964, 14, 17.

14. Levinsky, N. G., and R. C. Lalone. Sodium excretion during acute saline loading in dogs with vena caval constriction. J. clin. Invest. 1965, 44, 565.

15. Lichardus, B., and J. W. Pearce. Hemodynamic and humoral factors in natriuresis following blood volume expansion. Fed. Proc. 1965, 24, 404. 